\title{
Repercusión de la autoimagen en el diagnóstico de la capacidad de formar imágenes mentales
}

\author{
Alfredo Campos y M.a Angeles González \\ Universidad de Santiago de Compostela
}

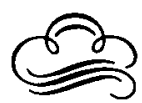

Resumen

Desde 1909 hasta la actualidad se han construido y utilizado mucho los cuestionarios para medir la capacidad de los sujetos para formar imágenes mentales; sin embargo, ban sido muy discutidos, fundamentalmente porque las autopuntuaciones de imagen están contaminadas por las expectativas sociales.

Nosotros estudiamos la relación de la autoestima y la autoimagen con la viveza subjetiva de imagen visual. Presentamos a 93 estudiantes de Formación Profesional un test objetivo de imagen, un test subjetivo de imagen, una medida de autoestima y una de autoimagen. Obtuvimos correlaciones significativas entre la autoestima y la medida subjetiva de imagen, $y$ entre la autoimagen y la medida subjetiva de imagen; sin embargo, la autoestima y la autoimagen no correlacionaron significativamente con un test objetivo de imagen.

Palabras clave: Imagen. Autoestima. Autoimagen.

\section{Abstract}

From 1909 until the present time, many questionnaires have been constructed and bave been often used to measure the ability of subjects to form mental imagery, bowever, they bave been strongly questioned, basically because imagery self-ratings are contaminated by response set to respond in a socially desirable manner.

We examine the relation of self-esteem and self-image with subjective vividness of visual imagery. We presented to 93 polytechnic students one objective test of imagery, one subjective test of imagery, one measure of sel-esteem, and one measure of self-image. We obtained significant correlations between self-esteem and the subjective measure of imagery vividness, and between self-image and the measure of imagery vividness; however, self-esteem and self-image did not correlate significantly with an objective test of imagery.

Key words: Imagery. Self-esteem. Self-image.

Dirección del autor: A. Campos. Universidad de Santiago. Dpto. Psicología Básica. Santiago de Compostela. 


\section{8}

El diagnóstico de la capacidad para formar imágenes se ha efectuado, en la reciente historia de las imágenes mentales, fundamentalmente a través de cuestionarios. Desde la publicación en 1909 por Betts, del «Questionnaire Upon Mental Imagery», se han construido otros con la finalidad de diagnosticar diferentes aspectos de las imágenes mentales. De entre todos ellos destacamos, por su utilización frecuente, el «Betts Questionnaire Upon Mental Imagery» de Sheehan (1967), que es una revisión y actualización del de Betts (1909). Otros bastante utilizados son el «Gordon's Imagery Control Scale» (Richardson, 1969), el «Individual Differences Questionnaire» (Paivio, 1971), el «Imagery Survey Schedule» (Cautela y Tondo, 1971), el «Vividness of Visual Imagery Questionnaire» (Marks, 1973), la «Visual Elaboration Scale» (Slee, 1976), el «VerbalizaerVisualizer Questionnaire» (Richardson, 1977a), y el «Vividness of Movement Imagery Questionnaire» (Isaac, Marks y Russell, 1986).

Como medidas objetivas de la capacidad de formar imágenes se han utilizado fundamentalmente cuatro test, el «Flags test» (Thurstone y Jeffrey, 1956), el «Minnesota Paper Form Board» (MPFG) (Likert y Quasha, 1941), la escala de Relaciones Espaciales del DAT (Bennett, Seashore y Wesman, 1947), y el Test Espacial del PMA (Thurstone, 1938).

Las pruebas subjetivas de imagen han sido más utilizadas que las objetivas en el diagnóstico de las habilidades de imagen. En un reciente artículo, Marks (1989) hace un balance del uso de su test, el «Vividness of Visual Imagery Questionnaire» (1973), desde 1972 hasta 1988, utilizando como fuente de información el «Psychological Abstracts» y el «Journal of Mental Imagery». El test fue traducido, además del inglés, al francés (Denis, 1982), al alemán (Bishof y Steiner, 1986), al japonés (Hishitani, 1985), al polaco (Winczo-Kostecka y Cielecki, 1985) y al español (Campos, 1988). Relata que en ese período se han contabilizado 108 trabajos experimentales y 42 críticas y discusiones sobre él. Los cuestionarios de imagen, a pesar de haber sido tan utilizados, han sido muy discutidos (White, Ashton y Law, 1978; Ashton y White, 1980; Kaufmann, 1981, 1983; Chara, 1989; Chara y Hamm, 1989), fundamentalmente porque se basan en autovaloraciones del individuo que pueden ser falseadas con facilidad.

Una de las críticas más fuertes que se hacen a los cuestionarios de imagen es que sus resultados están afectados por las expectativas de los individuos. Ya Woodworth, en 1910, comentando el test de Betts (1909), afirmaba que las puntuaciones del test podrían indicar simplemente diferencias entre los sujetos en su tendencia natural a clasificarse altos en imagen. Snyder (1972) encontró que el «Gordon's Test of Visual Imagery Control» (TVIC) (un test de autovaloración de imagen) correlacionaba moderada, pero significativamente, con dos medidas objetivas de imagen, la escala de Relaciones Espaciales del DAT $(r=.29)$, $y$ con el «Minnesota Paper Form Board» (MPFB) $(r=.22)$. Sin embargo, Ernest (1976) no encontró relación entre le test de Gordon (autopuntuación de imagen) y la escala de Relaciones Espaciales del DAT (test objetivo de imagen), ni entre el test de Gordon y el «Flags test» (test objetivo de imagen).

DiVesta, Ingersoll y Sunshine (1971) demuestran que existe una fuerte repercusión de las expectativas sociales en las puntuaciones de imagen cuando ésta se mide a través de un test de autovaloración, como es el «Betts Questionnaire Upon Mental Imagery». Durndell y Wettherick (1975) encontraron correlaciones significativas entre el «Betts Questionnaire Upon Mental Imagery» y una escala de expectativas sociales $(r=.46)$ en una muestra de sujetos. En otra muestra encontraron que los sujetos altos en control de imagen tenían significa- 
tivamente mayores puntuaciones en expectativas sociales que los sujetos que eran bajos en el control de imagen. Richardson (1977b) encontró que el «Questionnaire Upon Mental Imagery» y una escala de deseo social tenían una correlación positiva en los hombres $(r=.43)$, pero no en las mujeres. En cambio, otros estudios (Durndell y Wetherick, 1975; Ashton y White, 1975) no encontraron correlaciones significativas entre las escalas de autovaloración de imagen y las escalas de expectativas sociales.

En nuestra investigación deseamos averiguar si la autoestima y la autoimagen influyen en la puntuación de viveza de imagen, tanto cuando ésta se mide a través de cuestionarios como cuando se mide a través de pruebas objetivas.

\section{METODO}

Hemos utilizado como sujetos a 93 estudiantes de Formación Profesional (43 hombres y 53 mujeres), con una media de edad de 19,5 años, y con un rango que oscilaba entre 17 y 22 años. A todos los sujetos les hemos aplicado dos pruebas de medición de imagen, una objetiva, la escala Espacial del test de Aptitudes Mentales Primarias (PMA), y una subjetiva, el «Vividness of Visual Imagery Questionnaire» (Marks, 1973). Para medir la autoestima hemos utilizado la Escala de Autoestima de Guttman (Rosenberg, 1973), y para la valoración de la autoimagen como persona una escala de 6 puntos.

El «Vividness of Visual Imagery Questionnaire» consta de 16 ítems en los que se le pregunta a los sujetos sobre la claridad de sus imágenes. Ellos deben responder a cada ítem en una escala que oscila desde «perfectamente clara» (1), hasta «ninguna imagen» (5). Primero deben responder el cuestionario con los ojos abiertos y después con ellos cerrados. Las puntuaciones bajas indican viveza de imagen alta.

La Escala de Autoestima de Guttman (Rosenberg, 1973) consta de 6 ítems, alguno con varias preguntas. Los sujetos deben responder si están muy de acuerdo, de acuerdo, no están de acuerdo o muy en desacuerdo con lo que se pregunta en cada ítem. Las puntuaciones bajas indican alta autoestima.

La otra escala utilizada fue la de Autoimagen como personas, que constaba de un solo ítem que el sujeto debía contestar valorando desde uno (muy mala) hasta siete (muy buena) (Crowne, 1961; Gimeno Sacristán, 1976; Rodríguez Espinar, 1982).

Las pruebas se presentaron a grupos de aproximadamente veinte sujetos cada uno, en una única sesión, y se ha contrabalanceado el orden de presentación de las mismas.

\section{RESULTADOS}

La media y la desviación típica obtenidas por los sujetos en cada una de las escalas aparecen en la Tabla 1. También aparecen en la misma tabla las correlaciones de Pearson entre las puntuaciones obtenidas por los sujetos en las distintas escalas. 


\section{TABLA 1}

Correlaciones entre las distintas medidas

\begin{tabular}{lcccc}
\hline & Marks & PMA & Guttman & Autoimagen \\
\hline PMA & .06 & & & \\
Guttman & $.38^{\star}$ & -.12 & & \\
Autoimagen & $-.49^{*}$ & -.09 & $-.47^{*}$ & \\
X & 70.37 & 18.10 & 2.16 & 5.17 \\
Sx & 20.29 & 11.02 & 1.63 & 1.06 \\
\hline
\end{tabular}

$\mathrm{p}<.01$

Hemos encontrado correlaciones significativas entre la autoestima (Guttman) y la viveza subjetiva de imagen visual, y entre la autoimagen y la viveza subjetiva de imagen visual. Sin embargo, no hemos encontrado correlaciones significativas entre la autoestima (Guttman) y la viveza objetiva de imagen (PMA), ni entre la autoimagen y la viveza objetiva de imagen.

El segundo análisis que hemos efectuado fue averiguar si tanto en los hombres como en las mujeres se mantenían esas mismas correlaciones. Las correlaciones obtenidas por los hombres aparecen en la Tabla 2.

\section{TABLA 2}

Correlaciones de Pearson obtenidas por los hombres

\begin{tabular}{lcccc}
\hline & Marks & PMA & Guttman & Autoimagen \\
\hline PMA & -.05 & & & \\
Guttman & .25 & -.20 & & \\
Autoimagen & $-.40^{*}$ & -.14 & -.19 & \\
X & 68.18 & 22.32 & 1.96 & 5.32 \\
Sx & 16.56 & 10.83 & 1.48 & 1.02 \\
\hline
\end{tabular}

$\mathrm{p}<.01$

La autoestima (Guttman) no correlacionó significativamente con ninguna de las dos medidas de imagen; sin embargo, la autoimagen correlacionó significativamente con la viveza subjetiva de imagen visual, pero no correlacionó con la viveza objetiva de imagen. Las correlaciones obtenidas por las mujeres aparecen en la Tabla 3.

TABLA 3

Correlaciones de Pearson obtenidas por las mujeres

\begin{tabular}{lcccc}
\hline & Marks & PMA & Guttman & Autoimagen \\
\hline PMA & .19 & & & \\
Guttman & $.43^{*}$ & -.01 & & \\
Autoimagen & $-.52^{*}$ & -.15 & -.63 & \\
X & 72.11 & 14.71 & 2.31 & 5.06 \\
Sx & 22.93 & 10.10 & 1.75 & 1.08 \\
\hline
\end{tabular}

$\mathrm{p}<.01$ 
En las mujeres encontramos correlaciones significativas entre la autoestima (Guttman) y la viveza subjetiva de imagen visual, y entre la autoimagen y la viveza subjetiva de imagen visual; sin embargo, no encontramos correlaciones significativas de la autoimagen ni de la autoestima con la viveza objetiva de imagen.

El siguiente análisis consistió en seleccionar los sujetos bajos (puntuación en el test superior a 3) y altos en autoestima (Guttman) (puntuación en el test inferior a 3), y averiguar si existía diferencia entre ellos en viveza objetiva de imagen y en viveza subjetiva de imagen visual. Los sujetos de alta autoestima obtuvieron una media en viveza objetiva de imagen de $18.84, \mathrm{Sx}=11.06$, y los bajos, $x=15.23, \mathrm{Sx}=10.81$. La diferencia entre ambos grupos no fue significativa $(t=1.05, p>.05)$. En cambio, la diferencia entre estos mismos sujetos en viveza subjetiva de imagen visual (Marks) fue significativa $(t=-.3 .63, \mathrm{p}<.001)$. $\mathrm{La}$ media de viveza subjetiva de imagen visual de los bajos en autoestima (Guttman) fue de $87.0, \mathrm{~S} x=22.88$, y la de los altos fue de 66.04, $\mathrm{S} x=17.32$.

El último análisis consistió en averiguar si existía diferencia en viveza objetiva y subjetiva de imagen entre los altos (puntuación en el test superior a 4) y los bajos (puntuación en el test inferior a 4) en autoimagen. La media de imagen objetiva en los sujetos de baja autoimagen fue de $16.74, \mathrm{Sx}=10.56$, y la de los altos, de 21.00, $\mathrm{Sx}=11.70$. Al hallar la diferencia entre ambos grupos en viveza objetiva de imagen obtuvimos un $t=1.44, p>.05$ ). En cambio, sí obtuvimos diferencia significativa entre los bajos y altos en autoimagen en su viveza subjetiva de imagen visual $(\mathrm{t}=4.14, \mathrm{p}<.001)$. La media de viveza subjetiva de imagen visual de los sujetos con alta autoimagen fue de $84.20, \mathrm{Sx}=23.34$, y la de los bajos fue de 63.93, Sx $=15.09$.

\section{DISCUSION}

Hemos obtenido correlaciones significativas entre la autoestima y la viveza subjetiva de imagen visual, y entre la autoimagen y la viveza subjetiva de imagen visual; sin embargo, ni la autoimagen ni la autoestima correlacionaron significativamente con la viveza objetiva de imagen. Por otra parte, también encontramos diferencias en viveza subjetiva de imagen visual entre los altos y bajos en autoestima, y entre los altos y bajos en autoimagen, diferencias que no se encontraron en viveza objetiva de imagen.

Todos estos datos nos podrían llevar a la conclusión de que los sujetos se ven influenciados por su autoestima y por su autoconcepto cuando se puntúan en los cuestionarios de viveza subjetiva de imagen visual. Sin embargo, creemos que no se puede sacar esa conclusión si tenemos en cuenta la gran cantidad de estudios (ver Ernest, 1977, y White, Sheehan y Ashton, 1977, para una revisión) que demuestran que el «Vividness of Visual Imagery Questionnaire» es una buena medida de imagen, ya que sus puntuaciones correlacionan con el aprendizaje, la memoria, la percepción, la creatividad, la resolución de problemas, con otros cuestionarios de imagen y con medidas fisiológicas.

Las conclusiones que podemos sacar de esta investigación son: $10^{\circ}:$ El «Vividness of Visual Imagery Questionnaire» correlaciona de forma insignificante con el test Espacial del PMA. Ernest (1976), Richardson (1977a), Snyder (1972) y Edwards y Wilkins (1981) tampoco encontraron correlaciones significativas en- 
tre los cuestionarios de valoración de imagen y pruebas objetivas de imagen. La razón probablemente sea que la mayoría de los cuestionarios miden imagen visual, mientras que los test objetivos miden imagen espacial. 2. ${ }^{\circ}$ : La autoestima correlaciona significativamente con la viveza subjetiva de imagen visual en el grupo de mujeres y en el total; en cambio, no correlaciona significativamente con la viveza objetiva de imagen. $30^{\circ}:$ La autoestima correlaciona significativamente con la viveza subjetiva de imagen visual, tanto en los hombres como en las mujeres, y en el grupo total; sin embargo, no correlaciona significativamente con la viveza objetiva de imagen.

Creemos que es necesario efectuar nuevos estudios para averiguar si estas correlaciones que hemos efectuado se deben a que en realidad la viveza de imagen visual correlaciona con la autoimagen y la autoestima, o el cuestionario mide viveza de imagen visual, pero sus puntuaciones están falseadas por la autoestima y la autoimagen.

\section{Referencias}

Ashton, R., Y WHITE, K. (1975). The effects of instructions on subjects imagery questionnaire scores. Social Bebaviour and Personality, 3, 41-43.

Ashton, R., Y WhITE, K. D. (1980). Sex differences in imagery vividness: an artifact of the test. British Journal of Psychology, 71, 35-38.

Bennett, G. K.; Seashore, M. G., y Wesman, A. G. (1947). Differential Aptitude Tests. Nueva York: The Psychological Corporation.

Bishof, D., y StEINER, G. (1986). Visual imagery during central and peripheral distraction. En D. G. Russell, D. F. Marks y J. T. E. Richardson (Eds.). Imagery 2. Dunedin, Nueva Zelanda: Human Performance Associates.

Campos, A. (1988). Vividness of imagery and positive and negative values of emotionality of words. Perceptual and Motor Skills, 67, 433-434.

Cautela, J. R., y Tondo, T. R. (1971). Imagery Survey Schedule. (Cuestionario de imagen no publicado, Boston College.)

Chara, P. J. (1989). A questionable questionnaire: A rejoinder to Marks. Perceptual and Motor Skills, 68, 159-162.

Chara, P. J., y Hamm, D. A. (1989). An inquiry into the construct validity of the Vividness of Visual Imagery Questionnaire. Perceptual and Motor Skills, 69, 127-136.

CROWNE, D. P. (1961). Self-acceptance and self-evaluative behaviour: A critique of methodology. Psychological Bulletin, 58, 104-125.

DENIS, M. (1982). Imaging while reading text: a study of individual differences. Memory and Cognition, $10,540-545$.

Di Vesta, F.; InGERSOLL, G., y Sunshine, P. (1971). A factor analysis of imagery tests. Journal of Verbal Learning and Verbal Bebavior, 10, 471-479.

DuRndell, A. J., Y WeTHERICK, N. E. (1975). Reported imagery and social desirability. Perceptual and Motor Skills, 41, 987-992.

EDwards, J. E., y WILKINS, W. (1981). Verbalizer-Visualizer Questionnaire: Relationship with imagery an verbal-visual ability. Joumal of Mental Imagery, 5, 137-142.

ERNEST, C. H. (1976). Verbal and noverbal processing sustems in high and low imagers: A study of system differentiation. Manuscrito no publicado.

ERNEST, C. H. (1977). Imagery ability and cognition: A critical review. Joumal of Mental Imagery, 2, 181-216.

Gimeno SACRISTÁN, J. (1976). Autoconcepto, sociabilidad y rendimiento escolar. Madrid: INCIE.

Hishitani, S. (1985). Imagery differences and task characteristic in memory. In D. F. Marks y. D. G. Russell (Eds.). Imagery 1. Dunedin, Nueva Zelanda: Human Performance Associates.

IsAAC, A.; MARKS, D. F., y RuSELl, D. G. (1986). An instrument for assessing imagery of movement: the Vividness of Movement Imagery Questionnaire. Joumal of Mental Imagery, $10,23-30$. 
KaUfmann, G. (1981). What is wrong with imagery questionnaires? Scandianvian Joumal of Psychology, 22, 59-64.

Kaufmann, G. (1983). How good are imagery questionnaires? A rejoinder to David Marks. Scandianvian Joumal of Psychology, 24, 247-249.

Likert, R., y Quasha, W. H. (1941). Revised Minnesota Paper Form Board Test. Nueva York: The Psychological Corporation.

MARKS, D. F. (1973). Visual imagery differences in the recall of pictures. Bristish Journal of Psycbology, 64, 17-24.

MARKS, D. F. (1989). Bibliography of research utilizing the Vividness of Visual Imagery Questionnaire. Perceptual and Motor Skills, 69, 707-718.

PAIviO, A. (1971). Imagery and verbal processes. Nueva York: Holt, Rinehart and Winston.

Richardson, A. (1969). Mental imagery. London: Routledge and Kegan Paul.

Richardson, A. (1977a). Verbalizer-visualizer: A cognitive style dimension. Journal of Mental Imagery, 1, 109-126.

Richardson, A. (1977b). The meaning and measurement of memory imagery. British Journal of Psychology, 68, 29.43.

Rodriguez Espinar, S. (1982). Factores de rendimiento escolar. Barcelona: Oikos-tau.

RosenberG, M. (1973). La autoimagen del adolescente y la sociedad. Buenos Aires: Paidós.

SheEHAN, P. W. (1967). A shortened form of Betts' Questionnaire Upon Mental Imagery, Joumal of Clinical Psychology, 23, 386-389.

SLEE, J. A. (1976). The perceptual nature of visual imagery. Tesis Doctoral no publicada, Universidad Nacional de Camberra, Australia.

SNYDER, C. R. (1972). Individual differences in imagery and thought. Tesis Doctoral. Universidad de Oregón.

Thurstone, L. L. (1938). Primary Mental Abilities. Psychometrika Monographs, N. ${ }^{\circ} 1$.

Thurstone, L. L., y Jefrrey, T. E. (1956). Flags: A test of space thiking. Chicago: Industrial Relations Center.

VinCzo.Kostecka, M., y CieleCKI, M. (1985). Self-identity or self-esteem vivid and non-vivid imagers responses to threat to self-differentiation. Cabier de Psycbologie Cognitive, 5 , 171-186.

White, K. D.; Ashton, R., y Law, H. (1978). The measurement of imagery vividness: effects of format and order on the Betts Questionnaire Upon Mental Imagery. Canadian Joumal of Behavioural Science, 10, 68-78.

White, K.; SHEEHAN, P. W., y Ashton, R. (1977). Imagery assessment: A survey of self-report measures. Joumal of Mental Imagery, 1, 145-170.

Woodworth, R. S. (1910). Mental imagery. Psychological Bulletin, 7, 351-352. 